\title{
Dromostanolone Propionate
}

National Cancer Institute

\section{Source}

National Cancer Institute. Dromostanolone Propionate. NCI Thesaurus. Code C1077.

The propionate salt form of dromostanolone, a synthetic anabolic steroid related to dihydrotestosterone that has antiestrogenic effects. Dromostanolone inhibits the growth of estrogen receptor-presenting breast cancers; its virilizing effects limit its clinical usefulness. $(\mathrm{NCl})$ 